A. Rahbar-Ranji, Assoc. Prof., A. Shahbaztabar

AmirKabir University of Technology, Hafez Ave., Tehran, 15914, Iran, e-mail:rahbar@aut.ac.ir

\title{
FREE VIBRATION ANALYSIS OF BEAMS
}

\section{ON A PASTERNAK FOUNDATION USING LEGENDRE POLYNOMIALS AND RAYLEIGHT-RITZ METHOD}

\begin{abstract}
A. Rahbar-Ranji, A. Shahbaztabar. Аналіз вільних вібрацій балок на основі Пастернака з використанням полиномов Лежандра і метода Релея-Рітца. Досліджується вільна вібрація балок Ейлера-Бернуллі і Тимошенко, що спираються на двопараметричну пружню основу Пастернака. Для виведення керуючого рівняння використовується метод Релея-Рітца, а многочлени Лежандра, помножені на граничну функцію, використовується в якості допустимих функцій для визначення полів зсуву. Точність результатів оцінюється в порівнянні з даними, доступними в літературі. Показано, що метод має хорошу конвергенцію незалежно від теорії балок, граничних умов і параметрів пружної основи. Наведені природні частоти вібрації балок 3 різними граничними умовами, параметри пружної основи і відносини висоти до довжини.

Ключові слова:вільна вібрація, метод Релея-Рітца, теорія балок Ейлера-Бернуллі, теорія балок Тимошенко, пружня основа Пастернака

A. Rahbar-Ranji, A. Shahbaztabar. Free vibration analysis of beams on a Pasternak foundation using Legendre polynomials and Rayleigh-Ritz method. A free vibration of Euler-Bernoulli and Timoshenko beams resting on a two-parameter elastic foundation of Pasternak type has been investigated. Rayleigh-Ritz method is employed to deduce the governing equation and the Legendre polynomials multiplied by a boundary function is used as admissible functions to define the displacement fields. Accuracy of the results is evaluated by comparing with those available in the literature. It is shown that the method has a good and rapid convergence regardless of the beam theory, boundary conditions and elastic foundation parameters. Natural frequencies of beams with different boundary conditions, elastic foundation parameters, and ratios of height-to-length are presented.

Keywords: Free vibration; Rayleigh-Ritz method; Euler-Bernoulli’s beam theory; Timoshenko beam theory; Pasternak elastic foundation
\end{abstract}

Introduction. Beams and plates resting on elastic foundations have many applications in all branches of engineering. Building foundations, railroad ties, and engine foundations are some examples of beam/plate resting on elastic foundations. The simplest model of elastic foundation was introduced by Winkler [1]. In this model a vertical displacement of the foundation is assumed to be proportional to the contact pressure and the proportionality constant is called the modulus of foundation. In the Winkler type of elastic foundation there is no mutual interaction between adjacent springs. Different models have been introduced to consider this interaction. Pasternak [2] is a two-parameter elastic foundation that interaction is accomplished by connecting the ends of the springs to a shear layer.

Depending upon the ratio of height-to-length of beams, different theories are used to express the kinematic of deformation. For beams with a low ratio of height-to-length, Euler-Bernoulli Beam Theory (EBBT), in which shear deformation is ignored and planes normal to the longitudinal fibers remain plane and normal after deformation, should be used. For beams with a high ratio of height-to-length, Timoshenko Beam Theory (TBT), in which plane cross sections remain plane but not perpendicular to longitudinal, is used [3, 4].

Many investigations have been carried out to study free vibrations of both EBBs and TBs on an elastic foundation. Eisenberger and Clastornik [5] solved the eigenvalue problems of vibration and stability of an EBB resting on a variable elastic foundation. De Rosa [6] investigated the stability and dynamics of beams on a Winkler type elastic foundation by the cell discretization method. Zhou [7] presented a general solution to vibration of EBBs on the variable Winkler type of elastic foundations. Naidu and Rao [8] have studied the vibration of an uniform EBB initially stressed on Winkler and Pas-

DOI: .

(c) 2017 The Authors. This is an open access article under the CC BY license (http://creativecommons.org/licenses/by/4.0/). 
ternak types of foundation. Franciosi and Masi [9] have used the Finite Element Method (FEM) to study EBBs on elastic foundations of the variable Winkler type. Yokoyama and Suita [10] have obtained the natural frequencies and transient responses of TBs resting on a two-parameter elastic foundation using FEM. Yokoyama [11] has obtained the parametric instability of a TB resting on a Winkler type elastic foundation using FEM. Abbas and Thomas [12] have developed a FEM for stability analysis of TBs resting on an elastic foundation subjected to periodic axial loads.Thambiratnam and Zhuge [13] have used a simple FEM for free vibration analysis of EBBs on an elastic foundation. Balkaya et al. [14] have used the differential transform method to analyse the free vibration of EBBs/TBs resting on a elastic soil. Wang et al. [15] have presented an exact solution for TBs on various elastic foundations using Green's function.

In the present paper, a free vibration of EBBs/TBs resting on a Pasternak type elastic foundation has been investigated. Rayleigh-Ritz method is employed to derive the governing equation and the Legendre polynomials multiplied by a boundary function are used as admissible functions to study the free vibration. Convergence and applicability of the method are demonstrated through some numerical examples.

Theory and formulation.Let's assume a straight uniform beam of length $L$, depth $\mathrm{H}$ with a rectangular cross-section of unit width which has rested on an elastic foundation of Pasternak type (Fig. 1).

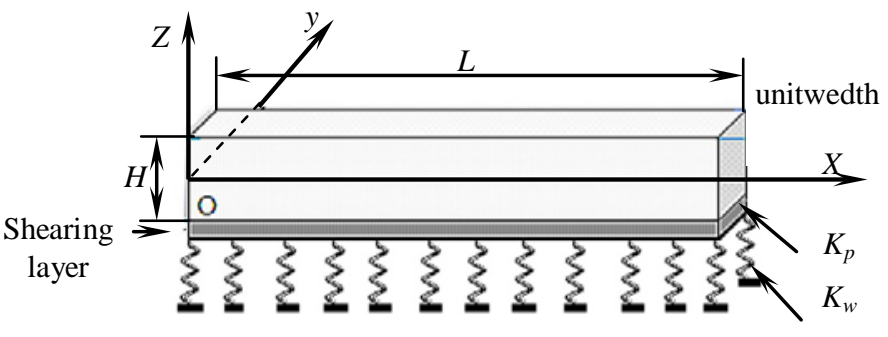

Fig. 1. A typical beam resting on an elastic foundation of Pasternak type

Displacement fields in $\mathrm{x}$ and $\mathrm{z}$ directions are defined as follows:

- EBBT:

$$
\begin{gathered}
u_{x}(x, z, t)=-z \frac{\partial w(x, t)}{\partial x}, \\
u_{z}(x, z, t)=w(x, t),
\end{gathered}
$$

- TBT:

$$
\begin{gathered}
u_{x}(x, z, t)=-z \psi(x, t), \\
u_{z}(x, z, t)=w(x, t),
\end{gathered}
$$

where $\psi(x, t)$ is the rotation function of the cross-section and $w(x, t)$ is transverse displacement function of the neutral axis. Stresses and strains are determined as follows:

- EBBT:

$$
\begin{gathered}
\varepsilon_{x}=-z \frac{\partial^{2} w(x, t)}{d x^{2}}, \\
\sigma_{x}=-E z \frac{\partial^{2} w(x, t)}{d x^{2}}, \\
\gamma_{x z}=0, \\
\tau_{x z}=0,
\end{gathered}
$$

- TBT:

$$
\varepsilon_{x}=-z \frac{\partial \psi(x, t)}{d x}
$$




$$
\begin{gathered}
\sigma_{x}=-E z \frac{\partial \psi(x, t)}{d x}, \\
\gamma_{x z}=\frac{\partial w(x, t)}{d x}-\partial \psi(x, t), \\
\tau_{x z}=K G \gamma_{x z},
\end{gathered}
$$

where $\varepsilon_{x}$ is the normal strain, $\sigma_{x}$ is the normal stress, $\gamma_{x z}$ is the shear strain, $\tau_{x z}$ is the shear stress, $E$ and $G$ are the Young's modulus and shear modulus, respectively, and $K$ is shear correction factor to account for non-uniform shear stress distribution.

The strain energy of a beam resting on Pasternak foundation is calculated as follows:

$$
U=\frac{1}{2} \int_{0}^{L} \int_{A}\left(\sigma_{x} \varepsilon_{x}+\tau_{x z} \gamma_{x z}\right) d A d x+\frac{k_{w}}{2} \int_{0}^{L}(w(x, t))^{2} d x+\frac{k_{p}}{2} \int_{0}^{L}\left(\frac{\partial w(x, t)}{\partial x}\right)^{2} d x,
$$

where $k_{w}$ and $k_{p}$ are Winkler and shear layer elastic coefficient of foundation, respectively. Substituting Eqs. (5)-(12) into (13) and integrating with respect to $z$, yields to following expression for calculation of the strain energy:

- EBBT:

$$
U^{E}=\frac{1}{2} \int_{0}^{L} E I\left(\frac{\partial w(x, t)}{\partial x}\right)^{2} d x+\frac{k_{w}}{2} \int_{0}^{L}(w(x, t))^{2} d x+\frac{k_{p}}{2} \int_{0}^{L}\left(\frac{\partial w(x, t)}{\partial x}\right)^{2} d x,
$$

- TBT:

$$
\begin{gathered}
U^{E}=\frac{1}{2} \int_{0}^{L}\left[E I\left(\frac{\partial \psi(x, t)}{\partial x}\right)^{2}+K A G\left(\frac{\partial \psi(x, t)}{\partial x}-\partial \psi(x, t)\right)^{2}\right] d x+ \\
+\frac{k_{w}}{2} \int_{0}^{L}(w(x, t))^{2} d x+\frac{k_{p}}{2} \int_{0}^{L}\left(\frac{\partial w(x, t)}{\partial x}\right)^{2} d x,
\end{gathered}
$$

which $I$ is the second moment of inertia and $A$ is the cross-section area. The kinetic energy of the beam is calculated as follows:

- EBBT:

$$
T^{E}=\frac{1}{2} \int_{0}^{L} \rho A\left(\frac{\partial w(x, t)}{\partial t}\right)^{2} d z
$$

- TBT:

$$
T^{T}=\frac{1}{2}\left[\int_{0}^{L} \rho A\left(\frac{\partial w(x, t)}{\partial t}\right)^{2}+\rho I\left(\frac{\partial \psi(x, t)}{\partial t}\right)^{2}\right] d x,
$$

where $\rho$ is the mass density of the beam. Following displacement functions are assumed:

$$
\begin{aligned}
& \psi(x, t)=\Psi(x) \cos \omega t, \\
& w(x, t)=W(x) \cos \omega t,
\end{aligned}
$$

where $\Psi(x)$ and $W(x)$ are mode shape functions and trigonometric term shows that the response of the free vibration is harmonic with natural frequency, $\omega$. Substituting Eqs. (18-19) into (14-17) yield to following expressions for maximum strain and kinetic energies:

- EBBT: 


$$
\begin{gathered}
U_{\max }^{E}=\frac{1}{2} \int_{0}^{L} E I\left(\frac{\partial^{2} W(x)}{\partial x^{2}}\right)^{2} d x+\frac{k_{w}}{2} \int_{0}^{L}(W(x, t))^{2} d x+\frac{k_{p}}{2} \int_{0}^{L}\left(\frac{\partial W(x)}{\partial x}\right)^{2} d x, \\
T_{\max }{ }^{T}=\frac{1}{2} \int_{0}^{L} \rho A(W(x)) d x,
\end{gathered}
$$

- TBT:

$$
\begin{gathered}
U_{\max }^{T}=\frac{1}{2} \int_{0}^{L}\left[E I\left(\frac{\partial \Psi(x, t)}{\partial x}\right)^{2}+K A G\left(\frac{\partial W(x, t)}{\partial x}-\partial \Psi(x, t)\right)^{2}\right] d x+ \\
+\frac{k_{w}}{2} \int_{0}^{L}(W(x, t))^{2} d x+\frac{k_{p}}{2} \int_{0}^{L}\left(\frac{\partial W(x, t)}{\partial x}\right)^{2} d x, \\
T_{\max }^{T}=\frac{\rho}{2} \int_{0}^{L} I \omega^{2}(\Psi(x))^{2}+A \omega^{2}(W(x))^{2} d x .
\end{gathered}
$$

For convenience, the following non-dimensional parameters are introduced:

$$
\begin{gathered}
\chi=\frac{x}{L}, \\
\bar{W}=\frac{W}{L}, \\
\bar{\Psi}=\Psi,
\end{gathered}
$$

The maximum total energy functional of the beam is defined as follows:

$$
\Pi=U_{\text {max }}-T_{\text {max }},
$$
lows:

Substituting Eqs. (24-26) into (20-23), total energy functional of the beam is obtained as fol- EBBT:

$$
\Pi^{E}=\frac{1}{2} \int_{0}^{L}\left(\frac{\partial^{2} \bar{W}}{\partial \chi^{2}}\right) d \chi-\frac{1}{2} \lambda^{4} \int_{0}^{L}(\bar{W}(\chi))^{2} d \chi+\frac{\bar{K}_{w}}{2} \int_{0}^{L}(\bar{W}(\chi))^{2} d \chi+\frac{\bar{K}_{p}}{2} \int_{0}^{L}\left(\frac{\partial \bar{W}(\chi)}{\partial \chi}\right)^{2} d \chi,
$$

- TBT:

$$
\begin{gathered}
\Pi^{T}=\frac{1}{2} \int_{0}^{L}\left[\left(\frac{\partial \bar{\Psi}(\chi)}{\partial \chi}\right)^{2}+\bar{K}\left(\frac{\partial \bar{W}(\chi)}{\partial \chi}-\bar{\Psi}(\chi)\right)^{2}\right] d \chi- \\
-\frac{1}{2} \lambda^{4} \int_{0}^{L}\left[\Gamma\left(\frac{\partial \bar{\Psi}(\chi)}{\partial \chi}\right)^{2}+(\bar{W}(\chi))^{2}\right] d \chi+\frac{\bar{K}_{w}}{2} \int_{0}^{L}(\bar{W}(\chi))^{2} d \chi+\frac{\bar{K}_{p}}{2} \int_{0}^{L}\left(\frac{\partial \bar{W}(\chi)}{\partial \chi}\right)^{2} d \chi
\end{gathered}
$$

where:$$
\bar{K}=\frac{K G A L^{2}}{E I},
$$$$
\lambda^{4}=\frac{\rho A \omega^{2} L^{2}}{E I},
$$$$
\Gamma=\frac{1}{2}\left(\frac{H}{L}\right)^{2},
$$ 


$$
\begin{aligned}
& \bar{K}_{w}=\frac{k_{w} L^{2}}{E I}, \\
& \bar{K}_{p}=\frac{k_{w} L^{2}}{E I \pi^{2}},
\end{aligned}
$$

where $\lambda$ is non-dimensional natural frequency, $\bar{K}_{w}$ and $\bar{K}_{p}$ are non-dimensional Winkler and shearing layer elastic coefficient of foundation, respectively. Following relation is held:

$$
\bar{K}=\frac{6 K}{(1+v)}\left(\frac{L}{H}\right)^{2} \text {. }
$$

Mode shape functions are assumed as follows:

- EBBT:

$$
\bar{W}(\chi)^{E}=f_{k} \sum_{l=1}^{N} A_{l} g_{l}(2 \chi-1),
$$

- TBT:

$$
\begin{aligned}
& \bar{\Psi}(\chi)^{E}=f_{u} \sum_{i=1}^{N} B_{l} g_{l}(2 \chi-1), \\
& \bar{W}(\chi)^{T}=f_{v} \sum_{j=1}^{N} C_{l} g_{l}(2 \chi-1),
\end{aligned}
$$

where $A_{l}, B_{i}$ and $C_{j}$ are the unknown constant coefficients, $g_{m}(2 \chi-1)(m=1,2,3, \ldots)$ is the onedimensional Legendre polynomial, $N$ is the number of Legendre polynomial terms, and $f_{s}=\chi^{r}(\chi-1)^{q}(s=k, u, v ; r, q=0,1,2)$ are the boundary functions which at least should satisfy the essential boundary conditions. Table 1 shows the selected parameters for simply supported (SS) and clamped (CC) boundary conditions. The Legendre polynomial series is a set of orthogonal series in the interval $0 \ldots 1$ which yields rapid convergence and more accuracy. Fig. 2 shows the first six terms of this polynomial.

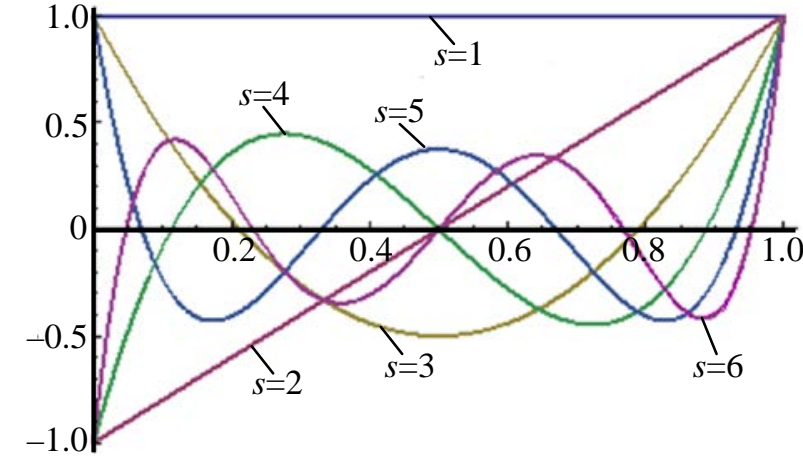

Fig. 2. The first six terms of the Legendre polynomials

Boundary function elements for different
boundary conditions
\begin{tabular}{c|c|c|c|c|c|c}
\hline \multirow{2}{*}{$\begin{array}{c}\text { Boundary } \\
\text { condition }\end{array}$} & \multicolumn{2}{|c|}{$f_{k}$} & \multicolumn{2}{|c|}{$f_{u}$} & \multicolumn{2}{|c}{$f_{v}$} \\
\cline { 2 - 8 } & $r$ & $q$ & $r$ & $q$ & $r$ & $q$ \\
\hline SS & 1 & 1 & 0 & 0 & 1 & 1 \\
\hline CC & 2 & 2 & 1 & 1 & 1 & 1 \\
\hline
\end{tabular}

The governing equation for free vibration analysis of EBB/TB is obtained by substituting Eqs. (36)-(38) into (28)-(29) and minimizing the total energy functional with respect to unknown coefficients as follows:

$$
\frac{\partial \Pi}{d A_{i}}=0 \frac{\partial \Pi}{d B_{i}}=0 \frac{\partial \Pi}{d C_{j}}=0 \quad l, i, j=1,2,3, \ldots, N .
$$

The generalized form of eigenvalue problem would be as follows:

$$
\left([k]-\lambda^{4}[m]\right)\{\Delta\}=0,
$$

where $k$ and $m$ are the stiffness and inertia matrices, respectively, $\Delta$ is the vector of unknown coefficients and $\lambda^{2}=\omega L^{2} \sqrt{\frac{\rho A}{E I}}$. 
Numerical and discussions. To demonstrate the convergence and applicability of the present method, free vibrations of EBB/TB are analyzed for different foundation parameters and boundary conditions. Table 2 gives convergence studies of current method for EBB with CC boundary condition and $\bar{K}_{w}, \bar{K}_{p}$. The number of Legendre polynomial terms has been increased from 2 to 18 . It can be seen that, for the first modes of vibration with two numbers of the Legendre polynomial terms, a very good result is achieved. For a higher mode of vibration, the number of the Legendre polynomial terms should be increased. For example, for fifth mode, $\mathrm{N}$ equal to 10 yields an accurate result.

Table 2

Convergence study of an EBB resting on a Pasternak foundation

(CC boundary condition, $\bar{K}_{w}, \bar{K}_{p}=2.5$ )

\begin{tabular}{c|c|c|c|c|c}
\hline \multirow{2}{*}{ № } & \multicolumn{5}{|c}{ Frequencyparameters } \\
\cline { 2 - 6 } & $\lambda_{1}$ & $\lambda_{2}$ & $\lambda_{3}$ & $\lambda_{4}$ & $\lambda_{5}$ \\
\hline 2 & 5.32010 & 8.4285 & - & - & - \\
\hline 4 & 5.32009 & 8.3825 & 11.6778 & 15.1898 & - \\
\hline 6 & 5.32003 & 8.3815 & 11.4368 & 14.5382 & 19.0306 \\
\hline 8 & 5.32003 & 8.3815 & 11.4281 & 14.4985 & 17.7129 \\
\hline 10 & 5.32003 & 8.3815 & 11.4280 & 14.4977 & 17.5908 \\
\hline 12 & 5.32003 & 8.3815 & 11.4280 & 14.4977 & 17.5862 \\
\hline 14 & 5.32003 & 8.3815 & 11.4280 & 14.4977 & 17.5861 \\
\hline
\end{tabular}

Table 3 shows convergence studies of the current method for TB with SS boundary conditions and $v=0.3, K=5 / 6, H / L=0.2, \bar{K}_{w}=10, \bar{K}_{p}=1$.In this case, an accurate result is achieved for the first mode of vibration, with $\mathrm{N}$ equal to four, and for the fifth mode of vibration - with $\mathrm{N}$ equal to 10 . It can be concluded that the current method has a good and rapid convergence irrespective of boundary conditions, beam theory and foundation parameters. For higher modes of vibration, more terms of the Legendre polynomial are needed.

Table 3

Convergence study of TB resting on Pasternak foundation

(SS boundary condition, $v=0.3, K=5 / 6, H / L=0.2, \bar{K}_{w}, \bar{K}_{p}$ )

\begin{tabular}{c|c|c|c|c|c}
\hline \multirow{2}{*}{ № } & \multicolumn{5}{|c}{ Frequency parameters } \\
\cline { 2 - 6 } & $\lambda_{1}$ & $\lambda_{2}$ & $\lambda_{3}$ & $\lambda_{4}$ & $\lambda_{5}$ \\
\hline 2 & 3.80593 & - & - & - & - \\
\hline 4 & 3.71567 & 6.54319 & 9.1318 & 11.8962 & - \\
\hline 6 & 3.71543 & 6.13311 & 8.2684 & 10.9306 & 13.0323 \\
\hline 8 & 3.71543 & 6.12573 & 8.2306 & 10.1212 & 11.8064 \\
\hline 10 & 3.71543 & 6.12570 & 8.2301 & 10.0342 & 11.6098 \\
\hline 12 & 3.71543 & 6.12570 & 8.2301 & 10.0313 & 11.5987 \\
\hline 14 & 3.71543 & 6.12570 & 8.2301 & 10.0313 & 11.5985 \\
\hline
\end{tabular}


Table 4 shows the first five natural frequencies of EBBs with SS boundary condition for different foundation parameters. The obtained results have been checked against Zhou [7] for the case of Winkler type of elastic foundation. Very good agreements can be seen regardless of foundation parameters and frequency number.

Table 4

Non-dimensional frequency parameters for EBB with SS boundary condition*

\begin{tabular}{|c|c|c|c|c|c|c|}
\hline \multicolumn{2}{|c|}{ Foundation parameters } & \multicolumn{5}{|c|}{ Frequency parameters } \\
\hline $\bar{K}_{w}$ & $\bar{K}_{p}$ & $\lambda_{1}$ & $\lambda_{2}$ & $\lambda_{3}$ & $\lambda_{4}$ & $\lambda_{5}$ \\
\hline \multirow{4}{*}{1} & 0 & 3.1496 & 6.28426 & 9.4251 & 12.5665 & 15.7080 \\
\hline & 0.5 & 3.4827 & 6.4718 & 9.5533 & 12.6635 & 15.7860 \\
\hline & 1 & 3.7408 & 6.6445 & 9.6766 & 12.7584 & 15.8628 \\
\hline & 2.5 & 4.3002 & 7.0947 & 10.0206 & 13.0310 & 16.0868 \\
\hline \multirow{4}{*}{10} & 0 & $\begin{array}{l}3.2193 \\
(3.220)\end{array}$ & $\begin{array}{c}6.2932 \\
(6.293)\end{array}$ & $\begin{array}{l}9.4277 \\
(9.427)\end{array}$ & $\begin{array}{l}12.5676 \\
(12.568)\end{array}$ & $\begin{array}{l}15.7086 \\
(15.709)\end{array}$ \\
\hline & 0.5 & 3.5347 & 6.4801 & 9.5560 & 12.6646 & 15.7865 \\
\hline & 1 & 3.7830 & 6.6522 & 9.6791 & 12.7595 & 15.8634 \\
\hline & 2.5 & 4.3282 & 7.1010 & 10.0229 & 13.0320 & 16.0873 \\
\hline \multirow{5}{*}{100} & \multirow{2}{*}{0} & 3.7484 & 6.3816 & 9.4545 & 12.5790 & 15.7144 \\
\hline & & $(3.748)$ & $(6.382)$ & $(9.454)$ & $(12.579)$ & (15.715) \\
\hline & 0.5 & 3.9608 & 6.5613 & 9.5816 & 12.6757 & 15.7923 \\
\hline & 1 & 4.1437 & 6.7273 & 9.7038 & 12.7703 & 15.8690 \\
\hline & 2.5 & 4.5824 & 7.1630 & 10.0451 & 13.0421 & 16.0927 \\
\hline \multirow{5}{*}{1000} & \multirow{2}{*}{0} & 5.7556 & 7.1121 & 9.7102 & 12.6905 & 15.7721 \\
\hline & & (5.755) & (7.112) & (9.710) & (12.690) & (15.773) \\
\hline & 0.5 & 5.8184 & 7.2438 & 9.8277 & 12.7847 & 15.8491 \\
\hline & 1 & 5.8793 & 7.3686 & 9.9412 & 12.8770 & 15.9250 \\
\hline & 2.5 & 6.0513 & 7.7095 & 10.2601 & 13.1424 & 16.1464 \\
\hline \multirow{4}{*}{10000} & 0 & 10.0243 & 10.3687 & 11.5652 & 13.6716 & 16.3167 \\
\hline & 0.5 & 10.0363 & 10.4122 & 11.6354 & 13.7472 & 16.3863 \\
\hline & 1 & 10.0483 & 10.4550 & 11.7043 & 13.8216 & 16.4551 \\
\hline & 2.5 & 10.0842 & 10.5806 & 11.9042 & 14.0378 & 16.6563 \\
\hline
\end{tabular}

Table 5 shows the first five natural frequencies of EBBs with CC boundary condition for different foundation parameters. The obtained results are examined against Franciosi and Masi [9], and very good agreements can be seen regardless of foundation parameters and frequency number.

\footnotetext{
* The numbers in the parentheses are taken from Zhou (1993)
} 
Table 5

Non-dimensional frequency parameters for an EBB with CC boundary conditions*

\begin{tabular}{|c|c|c|c|c|c|c|}
\hline \multicolumn{2}{|c|}{ Foundation parameters } & \multicolumn{5}{|c|}{ Frequency parameters } \\
\hline $\bar{K}_{w}$ & $\bar{K}_{p}$ & $\lambda_{1}$ & $\lambda_{2}$ & $\lambda_{3}$ & $\lambda_{4}$ & $\lambda_{5}$ \\
\hline \multirow{8}{*}{1} & 0 & 4.7324 & 7.8537 & 10.9958 & 14.1372 & 17.2788 \\
\hline & & $(4.73)$ & $(7.85)$ & $(11.0)$ & - & - \\
\hline & 0.5 & 4.8691 & 7.9683 & 11.0864 & 14.2116 & 17.3416 \\
\hline & & $(4.87)$ & $(7.97)$ & (11.09) & - & - \\
\hline & 1 & 4.9946 & 8.0780 & 11.1748 & 14.2847 & 17.4037 \\
\hline & & $(4.99)$ & $(8.08)$ & $(11.17)$ & - & - \\
\hline & 2.5 & 5.3200 & 8.3815 & 11.4280 & 14.4977 & 17.5861 \\
\hline & & $(5.32)$ & $(8.38)$ & $(11.43)$ & - & - \\
\hline \multirow{4}{*}{10} & 0 & 4.7535 & 7.8584 & 10.9975 & 14.1380 & 17.2792 \\
\hline & 0.5 & 4.8885 & 7.9728 & 11.0880 & 14.2124 & 17.3420 \\
\hline & 1 & 5.0125 & 8.0822 & 11.1765 & 14.2855 & 17.4041 \\
\hline & 2.5 & 5.3350 & 8.3853 & 11.4295 & 14.4984 & 17.5865 \\
\hline \multirow{8}{*}{100} & 0 & 4.9504 & 7.9043 & 11.0143 & 14.1460 & 17.2836 \\
\hline & & (4.95) & $(7.90)$ & $(11.01)$ & - & - \\
\hline & 0.5 & 5.0707 & 8.0168 & 11.1045 & 14.2202 & 17.3463 \\
\hline & & (5.23) & (8.16) & (11.24) & - & - \\
\hline & 1 & 5.1823 & 8.1245 & 11.1925 & 14.2932 & 17.4084 \\
\hline & & (5.54) & (8.39) & (11.43) & - & - \\
\hline & 2.5 & 5.4773 & 8.4232 & 11.4446 & 14.5058 & 17.5907 \\
\hline & & (5.48) & $(8.42)$ & $(11.44)$ & - & - \\
\hline \multirow{4}{*}{1000} & 0 & 6.2239 & 8.3251 & 11.1790 & 14.2248 & 17.3270 \\
\hline & 0.5 & 6.2857 & 8.4218 & 11.2653 & 14.2978 & 17.3893 \\
\hline & 1 & 6.3455 & 8.5150 & 11.3497 & 14.3696 & 17.4509 \\
\hline & 2.5 & 6.5136 & 8.7768 & 11.5918 & 14.5790 & 17.6319 \\
\hline \multirow{8}{*}{10000} & 0 & 10.1228 & 10.8392 & 12.5260 & 14.9493 & 17.7442 \\
\hline & & $(10.12)$ & $(10.84)$ & (12.53) & - & - \\
\hline & 0.5 & 10.1374 & 10.8835 & 12.5876 & 15.0122 & 17.8022 \\
\hline & & (10.16) & (10.94) & (12.68) & - & - \\
\hline & 1 & 10.1518 & 10.9272 & 12.6483 & 15.0744 & 17.8597 \\
\hline & & $(10.21)$ & $(11.04)$ & $(12.81)$ & - & - \\
\hline & 2.5 & 10.1943 & 11.0546 & 12.8252 & 15.2564 & 18.0287 \\
\hline & & $(10.41)$ & (11.38) & $(13.21)$ & - & - \\
\hline
\end{tabular}

Tables 6-9 depict the first five frequencies of TBs resting on a Pasternak elastic foundation with SS/CC boundary conditions. In these tables the number of Legendre polynomial terms is taken as 14 and different ratios of height-to-length are considered. It can be concluded that for height-to-length ratios less than 0.05 , this ratio has no influence on the results. However, the height-to-length ratio has an influence on the result when it is high. Its influence depends on boundary conditions, the mode number and foundation parameters. For example, when $\bar{K}_{w}=1$, for SS boundary conditions, the first natural frequency decreases by 1.5 percent when the height-to-length ratio increases from 0.002 to 0.2 . However, for CC boundary conditions and $\bar{K}_{p}=0$, it decreases about $8 \%$ and, for $\bar{K}_{p}=2.5$, it decreases by $10 \%$. Figure 3 shows the effect of the height-to-length ratio on the natural frequencies for the case $\bar{K}_{w}=100, \bar{K}_{p}=0.5$ and SS boundary condition. It can be seen that the effect of the height-

\footnotetext{
* The numbers in the parentheses are taken from Franciosi and Masi (1993)
} 
to-length ratio is more prominent for a higher mode of vibration, and when the ratio of height-tolength is less than 0.05, EBBT yields very accurate results.

Table 6

Non-dimensional frequency parameters for a TB with SS boundary conditions,

$$
\left(\bar{K}_{w}=1, v=0.3, K=5 / 6 \text {, and } N=14\right)
$$

\begin{tabular}{c|c|c|c|c|c|c}
\hline \multirow{3}{*}{$\begin{array}{c}\text { Height-to- } \\
\text { lengthratio }\end{array}$} & $\begin{array}{c}\text { Foundation } \\
\text { parameters }\end{array}$ & \multicolumn{5}{|c}{ Frequency parameters } \\
\cline { 2 - 7 } & $\bar{K}_{p}$ & $\lambda_{1}$ & $\lambda_{2}$ & $\lambda_{3}$ & $\lambda_{4}$ & $\lambda_{5}$ \\
\hline \multirow{3}{*}{0.002} & 0 & 3.1496 & 6.2841 & 9.4248 & 12.5658 & 15.7067 \\
\cline { 2 - 7 } & 0.5 & 3.4826 & 6.4718 & 9.5530 & 12.6628 & 15.7847 \\
\cline { 2 - 7 } & 1 & 3.7408 & 6.6444 & 9.6763 & 12.7577 & 15.8615 \\
\cline { 2 - 7 } & 2.5 & 4.3001 & 7.0946 & 10.0204 & 13.0303 & 16.0855 \\
\hline \multirow{3}{*}{0.05} & 0 & 3.1430 & 6.2324 & 9.2557 & 12.1815 & 14.9927 \\
\cline { 2 - 7 } & 0.5 & 3.4772 & 6.4231 & 9.3887 & 12.2850 & 15.0790 \\
\cline { 2 - 7 } & 1 & 3.7360 & 6.5982 & 9.5164 & 12.3861 & 15.1637 \\
\hline \multirow{3}{*}{0.10} & 0 & 4.2960 & 7.0535 & 9.8713 & 12.6752 & 15.4098 \\
\cline { 2 - 7 } & 0.5 & 3.1238 & 6.0917 & 8.8409 & 11.3433 & 13.6132 \\
\cline { 2 - 7 } & 1 & 3.7218 & 6.4737 & 9.1286 & 11.5850 & 13.8306 \\
\hline \multirow{3}{*}{0.20} & 2.5 & 4.2841 & 6.9442 & 9.5149 & 11.9213 & 14.1384 \\
\cline { 2 - 7 } & 0 & 3.0540 & 5.6722 & 7.8400 & 9.6573 & 11.2222 \\
\cline { 2 - 7 } & 0.5 & 3.4048 & 5.9072 & 8.0403 & 9.8487 & 11.4145 \\
\cline { 2 - 7 } & 1 & 3.6720 & 6.1165 & 8.2264 & 10.0292 & 11.5972 \\
\hline
\end{tabular}

Non-dimensional frequency parameters for a TB with SS boundary conditions,

$\left(\bar{K}_{w}=10, v=0.3, K=5 / 6\right.$, and $\left.N=14\right)$

\begin{tabular}{|c|c|c|c|c|c|c|}
\hline \multirow{2}{*}{$\begin{array}{l}\text { Height-to- } \\
\text { lengthratio }\end{array}$} & \multirow{2}{*}{$\begin{array}{c}\begin{array}{c}\text { Foundation } \\
\text { parameters }\end{array} \\
\bar{K}_{p}\end{array}$} & \multicolumn{5}{|c|}{ Frequency parameters } \\
\hline & & $\lambda_{1}$ & $\lambda_{2}$ & $\lambda_{3}$ & $\lambda_{4}$ & $\lambda_{5}$ \\
\hline \multirow{4}{*}{0.002} & 0 & 3.2193 & 6.29315 & 9.4275 & 1.5670 & 15.7073 \\
\hline & 0.5 & 3.5347 & 6.4801 & 9.5556 & 12.6640 & 15.7852 \\
\hline & 1 & 3.7830 & 6.6521 & 9.6788 & 12.7588 & 15.8620 \\
\hline & 2.5 & 4.3282 & 7.1010 & 10.0226 & 13.0313 & 16.0861 \\
\hline \multirow{4}{*}{0.05} & 0 & 3.2130 & 6.2416 & 9.2585 & 12.1827 & 14.9933 \\
\hline & 0.5 & 3.5294 & 6.4315 & 9.3914 & 12.2862 & 15.0796 \\
\hline & 1 & 3.7783 & 6.6059 & 9.5189 & 12.3872 & 15.1643 \\
\hline & 2.5 & 4.3241 & 7.0599 & 9.8736 & 12.6763 & 15.4104 \\
\hline \multirow{4}{*}{0.10} & 0 & 3.1946 & 6.1014 & 8.8440 & 11.3447 & 13.6141 \\
\hline & 0.5 & 3.5140 & 6.3002 & 8.9911 & 11.4674 & 13.7240 \\
\hline & 1 & 3.7644 & 6.4818 & 9.1314 & 11.5863 & 13.8314 \\
\hline & 2.5 & 4.3122 & 6.9507 & 9.5174 & 11.9225 & 14.1391 \\
\hline \multirow{4}{*}{0.20} & 0 & 3.1281 & 5.6843 & 7.8443 & 9.6596 & 11.2236 \\
\hline & 0.5 & 3.4590 & 5.9174 & 8.0442 & 9.8509 & 11.4159 \\
\hline & 1 & 3.7154 & 6.1257 & 8.2301 & 10.0313 & 11.5985 \\
\hline & 2.5 & 4.2710 & 6.6480 & 8.7217 & 10.5192 & 12.0978 \\
\hline
\end{tabular}


Non-dimensional frequency parameters for a TB with CC boundary conditions,

$\left(\bar{K}_{w}=1, v=0.3, K=5 / 6\right.$, and $\left.N=14\right)$

\begin{tabular}{c|c|c|c|c|c|c}
\hline \multirow{2}{*}{$\begin{array}{c}\text { Height-to- } \\
\text { length ratio }\end{array}$} & $\begin{array}{c}\text { Foundation } \\
\text { parameters }\end{array}$ & \multicolumn{5}{|c}{ Frequency parameters } \\
\cline { 2 - 7 } & $\bar{K}_{p}$ & $\lambda_{1}$ & $\lambda_{2}$ & $\lambda_{3}$ & $\lambda_{4}$ & $\lambda_{5}$ \\
\hline \multirow{4}{*}{0.002} & 0 & 4.7323 & 7.8535 & 10.9952 & 14.1360 & 17.2767 \\
\cline { 2 - 7 } & 0.5 & 4.8691 & 7.9681 & 11.0858 & 14.2103 & 17.3395 \\
\cline { 2 - 7 } & 1 & 4.9945 & 8.0777 & 11.1742 & 14.2835 & 17.4016 \\
\cline { 2 - 7 } & 2.5 & 5.3191 & 8.3813 & 11.4274 & 14.4965 & 17.5841 \\
\hline \multirow{4}{*}{0.05} & 0 & 4.6923 & 7.7041 & 10.6403 & 13.4612 & 16.1591 \\
\cline { 2 - 7 } & 0.5 & 4.8305 & 7.8222 & 10.7363 & 13.5426 & 16.2307 \\
\hline \multirow{5}{*}{0.10} & 1 & 4.9570 & 7.9350 & 10.8296 & 13.6226 & 16.3014 \\
\hline & 2.5 & 5.2840 & 8.2457 & 11.0957 & 13.8541 & 16.4463 \\
\hline & 0 & 4.5821 & 7.3318 & 9.8564 & 12.1455 & 14.2325 \\
\hline & 0.5 & 4.7250 & 7.611 & 9.9687 & 12.2484 & 14.3303 \\
\hline \multirow{4}{*}{0.20} & 2.5 & 4.8548 & 7.5835 & 10.0773 & 12.3487 & 14.4260 \\
\hline & 0 & 5.1875 & 7.9172 & 10.3830 & 12.6358 & 14.7024 \\
\hline & 0.5 & 4.2452 & 6.4188 & 8.2857 & 9.9040 & 11.3489 \\
\hline & 1 & 4.4087 & 6.5930 & 8.4594 & 10.0819 & 11.5325 \\
\hline & 2.5 & 4.5542 & 6.7539 & 8.6230 & 10.2505 & 11.7070 \\
\hline
\end{tabular}

Table 9

Non-dimensional frequency parameters for a TB with CC boundary conditions,

$\left(\bar{K}_{w}=10, v=0.3, K=5 / 6\right.$, and $\left.N=14\right)$

\begin{tabular}{c|c|c|c|c|c|c}
\hline \multirow{3}{*}{$\begin{array}{c}\text { Height-to- } \\
\text { lengthratio }\end{array}$} & $\begin{array}{c}\text { Foundation } \\
\text { parameters }\end{array}$ & \multicolumn{5}{|c}{ Frequency parameters } \\
\cline { 2 - 7 } & $\bar{K}_{p}$ & $\lambda_{1}$ & $\lambda_{2}$ & $\lambda_{3}$ & $\lambda_{4}$ & $\lambda_{5}$ \\
\hline \multirow{3}{*}{0.002} & 0 & 4.7534 & 7.8581 & 10.9969 & 14.1368 & 17.2771 \\
\cline { 2 - 7 } & 0.5 & 4.8885 & 7.9725 & 11.0875 & 14.2111 & 17.3399 \\
\cline { 2 - 7 } & 1 & 5.0125 & 8.0820 & 11.1759 & 14.2843 & 17.4020 \\
\cline { 2 - 7 } & 2.5 & 5.3349 & 8.3851 & 11.4289 & 14.4972 & 17.5845 \\
\cline { 2 - 7 } & 0 & 4.7139 & 7.7089 & 10.6422 & 13.4621 & 16.1596 \\
\cline { 2 - 7 } & 0.5 & 4.8503 & 7.8269 & 10.7381 & 13.5435 & 16.2312 \\
\cline { 2 - 7 } & 1 & 4.9753 & 7.9394 & 10.8314 & 13.6234 & 16.3020 \\
\cline { 2 - 7 } & 2.5 & 5.2991 & 8.2496 & 11.0973 & 13.8550 & 16.5084 \\
\cline { 2 - 7 } & 0 & 4.6051 & 7.3374 & 9.8586 & 12.1466 & 14.2332 \\
\cline { 2 - 7 } & 0.5 & 4.7460 & 7.4664 & 9.9709 & 12.2496 & 14.3310 \\
\hline \multirow{3}{*}{0.10} & 1 & 4.8742 & 7.5886 & 10.07942 & 12.3499 & 14.4267 \\
\cline { 2 - 7 } & 2.5 & 5.2035 & 7.9216 & 10.3850 & 12.6368 & 14.7030 \\
\cline { 2 - 7 } & 0 & 4.2738 & 6.4269 & 8.2894 & 9.9061 & 11.3503 \\
\cline { 2 - 7 } & 0.5 & 4.4343 & 6.6005 & 8.4629 & 10.0839 & 11.5337 \\
\cline { 2 - 7 } & 1 & 4.5775 & 6.7608 & 8.6262 & 10.2524 & 11.7083 \\
\hline
\end{tabular}


Fig. 4 shows the effect of elastic foundation parameters on the natural frequency of an EBB with CC boundary conditions. As can be seen, Winkler elastic foundation parameter has no effect on the natural frequency when it is less than 100 , however it has a significant effect for higher values. The effect of shearing parameter of foundation is the same for all values of $\bar{K}_{w}$.

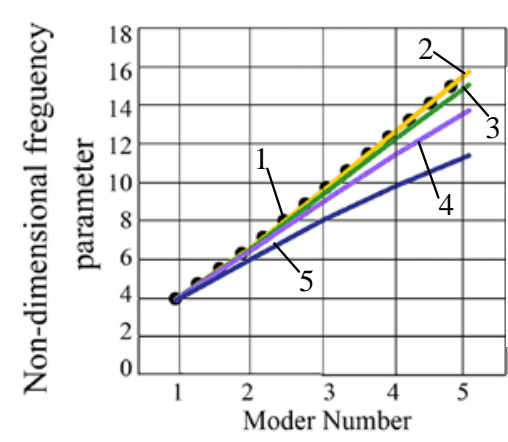

Fig. 3. The effect of height-to-length ratio on natural frequencies (SS boundary conditions, $\bar{K}_{w}=100$, $\left.\bar{K}_{p}=0.5\right): \operatorname{EBB}(1) ; H / L=0.02(2)$; 0.05 (3); 0.1 (4); 0.2 (5)

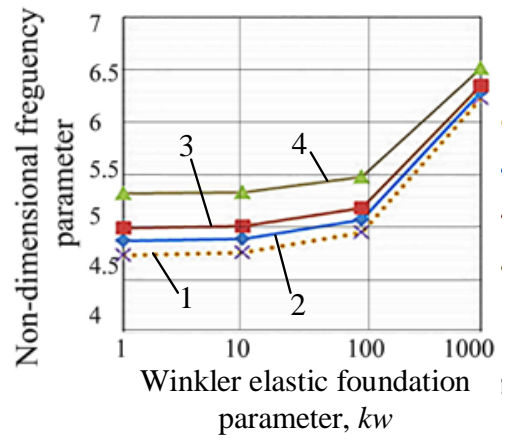

Fig. 4. The effect of elastic foundation parameter on the natural frequency of an $E B B$ (CC boundary conditions): $k p=0$ (1); 0.5 (2); 1.0 (3); 2.5 (4)

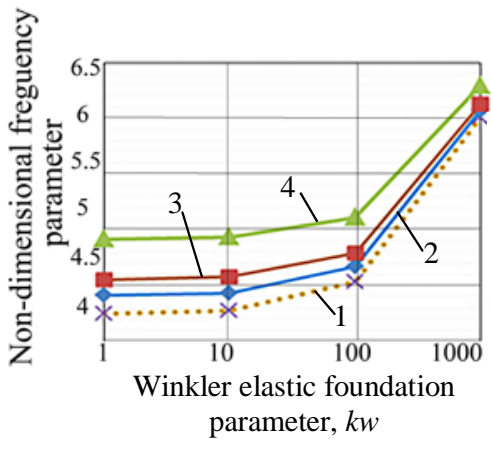

Fig. 5. The effect of elastic foundation parameter on the natural frequency of a TB (CC boundary conditions, $H / L=0.2)$ : $k p=0$ (1); 0.5 (2); 1.0 (3); 2.5 (4)

Conclusions. In the present paper, the free vibration of EBs/TBs resting on a Pasternak foundation is investigated. Rayleigh-Ritz method is used to obtain the governing equation and the Legendre polynomials multiplied by a boundary function is used as admissible functions. It is shown that the method has a very good and rapid convergence regardless of boundary conditions, beam theory and elastic foundation. Using different theories, the first five eigenvalues of beam on an elastic foundation are tabulated for different Winkler and shearing layer coefficients of foundation and the height-tolength ratios. It is observed that the results of the EBT/TBT for small ratios of the height-to-length are very close. However, as this ratio increases, the results of the EBBT and TBT are deviate depending upon boundary conditions, foundation parameter and the mode number. For a higher mode number, the effect of the height-to-length ratio is more prominent. The effects of elastic foundation parameters have been studied, and it is found that shearing parameter of foundation has no significant effect on the natural frequency of EBBs/TBs.

\section{Література}

1. Winkler E. Die Lehre von der Elasticitaet und Festigkeit, Dominicus. Prague. 1867.

2. Пастернак П.Л. Основы нового метода расчета фундаментов на упругом основании при помощи двух коэффициентов постели. Москва: Стройиздат, 1954, 56 с.

3. Timoshenko S.P. On the correction for shear of the differential equations for transverse vibration of prismatic bars. Philosophical Magazine, 1921, №41. 744-746.

4. Timoshenko S.P. On the transverse vibrations of bars of uniform cross-sections. Philosophical Magazine. 1922.№43. 125-131.

5. Eisenberger M., Clastornik J. Vibration and buckling of a beam on a variable Winkler elastic foundation. Journal of Sound and Vibration, 1987, №115, 233-241.

6. De Rosa M.A. (1989). Stability and dynamics of beams on Winkler elastic foundations. Earthquake engineering and Structural dynamics, 1989, №18, 377-388.

7. Zhou D. (1993). A general solution to vibrations of beams on variable Winkler elastic foundation. Computers and Structures. 1993.№47, 83-90.

8. Naidu N.R., Rao G.V. (1995). Vibrations of initially stressed uniform beams on a two-parameter elastic foundation. Computers and Structures, 1995, №57, 941-943. 
9. Franciosi C., Masi A. Free vibration of foundation beams on two-parameter elastic soil. Computers and Structures, 1993, №47, 419-426.

10. Yokoyama T. Suita, (1987). Vibrations and transient responses of Timoshenko beams resting on elastic foundation. Ingenieur-Archiv. 1987. №57, 81-90.

11. Yokoyama T. Parametric instability of Timoshenko beams resting on an elastic foundation. Computers and Structures. 1988. №28m 207-216.

12. Abbas B.A.H., Thomas J. Dynamic stability of Timoshenko beams resting on an elastic foundation. Journal of Sound and Vibration, 1987, №60, 33-44.

13. Thambiratnam D., Zhuge Y. Free vibration analysis of beams on elastic foundation. Computers and Structures, 1996. №60, 971-980.

14. Balkaya M., Kaya M.O., Saglamer A. Analysis of the vibration of an elastic beam supported on elastic soil using the differential transform method. Arch ApplMech, 2009, №79, 135-146.

15. Wang C.M., Lam K.Y., He X.Q. Exact solution for Timoshenko beams on elastic foundation using Green’s functions. Mechanical structures and Machines. 1998. №26. 101-113.

\section{Reference}

1. Winkler, E. (1867). Die Lehre von der Elasticitaet und Festigkeit, Dominicus. Prague.

2. Pasternak, P.L. (1954). On a new method of analysis of an elastic foundation by means of two foundation constants (in Russian), Gosudarstrennoe Izdatelstvo Literaturi po Stroitelstvu I Arkhitekture.

3. Timoshenko, S.P. (1921). On the correction for shear of the differential equations for transverse vibration of prismatic bars. Philosophical Magazine, 41, 744-746.

4. Timoshenko, S.P. (1922). On the transverse vibrations of bars of uniform cross-sections. Philosophical Magazine, 43, 125-131.

5. Eisenberger, M., \&Clastornik, J. (1987).Vibration and buckling of a beam on a variable Winkler elastic foundation. Journal of Sound and Vibration, 115, 233-241.

6. De Rosa, M.A. (1989). Stability and dynamics of beams on Winkler elastic foundations. Earthquake engineering and Structural dynamics, 18, 377-388.

7. Zhou, D. (1993). A general solution to vibrations of beams on variable Winkler elastic foundation. Computers and Structures, 47, 83-90.

8. Naidu, N.R., \& Rao, G.V. (1995). Vibrations of initially stressed uniform beams on a two-parameter elastic foundation. Computers and Structures, 57, 941-943.

9. Franciosi, C., \& Masi, A. (1993). Free vibration of foundation beams on two-parameter elastic soil. Computers and Structures, 47, 419-426.

10. Yokoyama, T., \& Suita, (1987). Vibrations and transient responses of Timoshenko beams resting on elastic foundation. Ingenieur-Archiv, 57, 81-90.

11. Yokoyama, T. (1988). Parametric instability of Timoshenko beams resting on an elastic foundation. Computers and Structures, 28m 207-216.

12. Abbas, B.A.H., \& Thomas, J. (1987). Dynamic stability of Timoshenko beams resting on an elastic foundation. Journal of Sound and Vibration, 60, 33-44.

13. Thambiratnam, D., \& Zhuge, Y. (1996). Free vibration analysis of beams on elastic foundation. Computers and Structures, 60, 971-980.

14. Balkaya, M., Kaya, M.O., \& Saglamer, A. (2009). Analysis of the vibration of an elastic beam supported on elastic soil using the differential transform method. Arch ApplMech, 79, 135-146.

15. Wang, C.M., Lam, K.Y., \& He, X.Q. (1998). Exact solution for Timoshenko beams on elastic foundation using Green’s functions. Mechanical structures and Machines, 26, 101-113. 